\title{
Narrative review of the thoracic endovascular aortic repair-first approach for mesenteric malperfusion in type A aortic dissection
}

\author{
Kanika Kalra, Bradley G. Leshnower, Edward P. Chen \\ Division of Cardiothoracic Surgery, Department of Surgery, Emory University School of Medicine, Atlanta, GA, USA \\ Contributions: (I) Conception and design: All authors; (II) Administrative support: None; (III) Provision of study materials or patients: BG Leshnower, \\ EP Chen; (IV) Collection and assembly of data: All authors; (V) Data analysis and interpretation: All authors; (VI) Manuscript writing: All authors; \\ (VII) Final approval of manuscript: All authors. \\ Correspondence to: Edward P. Chen, MD. Professor of Surgery, Division of Cardiothoracic Surgery, Department of Surgery, Emory University School \\ of Medicine, Atlanta, GA, USA. Email: edward.p.chen@emory.edu.
}

\begin{abstract}
The presence of malperfusion syndrome in type A aortic dissection (TAAD) is associated with a difficult and delayed diagnosis and high mortality. Current treatment paradigms have recently shifted towards correction of malperfusion followed by delayed proximal aortic repair approach for this challenging situation. This approach is based on the hypothesis that early restoration of blood flow to visceral tissue beds could prevent the lethal cascade of events leading to a necrotic bowel, persistent acidosis, and death. Several open and endovascular techniques have been described to address this treacherous complication. The objective of this work is to review the thoracic endovascular aortic repair (TEVAR)-first hybrid approach adopted at Emory University, in which a thoracic endograft is deployed in the true lumen first to restore adequate inflow to the relevant branch vessels. After successful reperfusion, aggressive volume resuscitation is adopted with the goal to correct metabolic acidosis under guarded conditions, while maintaining normotension for 24 hours. Proximal aortic repair is then performed using hypothermic circulatory arrest. This approach is suitable for hemodynamically stable patients with clinical and radiographic evidence of mesenteric ischemia and has shown improved outcomes with lower mortality rates, as compared to the conventional approach of emergent ascending aortic repair followed by laparotomy to address the presence of ischemic bowel.
\end{abstract}

Keywords: Type A aortic dissection (TAAD); mesenteric malperfusion; malperfusion syndrome; thoracic endovascular aortic repair (TEVAR)

Received: 12 August 2020; Accepted: 11 November 2020; Published: 20 January 2022.

doi: $10.21037 /$ jovs-20-155

View this article at: http://dx.doi.org/10.21037/jovs-20-155

\section{Introduction}

Type A aortic dissection (TAAD) is a catastrophic condition requiring emergent surgical intervention. Mortality following operative treatment remains high despite the best advance in surgical therapy and intraoperative as well as postoperative management, and is influenced by the preoperative patient state and other co-morbidities as suggested by the International Registry of Acute Aortic Dissection (IRAD) data (1). Around a quarter of patients with TAAD present with distal malperfusion which results from the intimal flap causing a partial or complete obstruction of the aorta or one if its side branches $(2,3)$. This obstruction can be static, from extension of dissecting flap into the branches; or from false lumen thrombosis; or dynamic from true lumen compression by false lumen expansion during each heartbeat. Such obstruction ultimately leads to malperfusion as a result of diminished blood flow to the arterial bed of a vital organ as demonstrated radiologically, and malperfusion syndrome which occurs secondary to diminished flow causing ischemia and impairment of end-organ function (4). The syndrome is characterized clinically by pain, neurologic symptoms or chemically by rising lactate or creatinine levels 


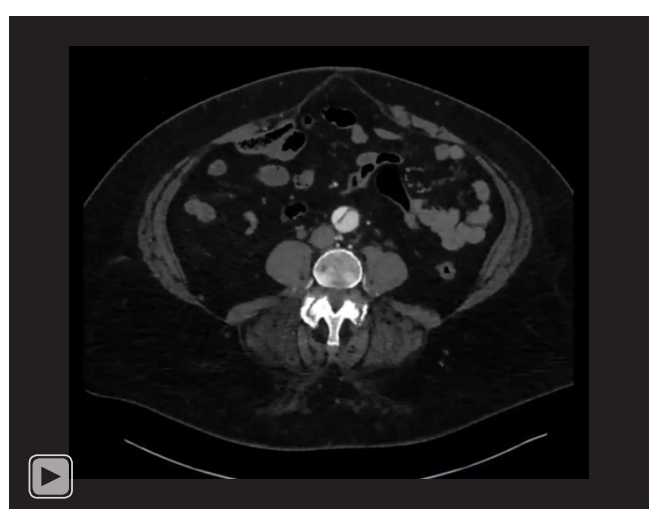

Video 1 A review of TEVAR-first approach for mesenteric malperfusion in TAAD.

worsening metabolic acidosis.

The incidence of visceral or mesenteric malperfusion in TAAD is about $3-6 \%(1-3)$ and is defined as any radiologic evidence of decreased perfusion through the celiac trunk, superior mesenteric artery (SMA), and inferior mesenteric artery with decreased viability or necrosis of the gut, with or without lactic acidosis, pain, or abdominal distention (1). It is known to be the most treacherous and deadliest complication associated with approximately 40-70\% mortality depending upon the series (5-7). Such abysmal outcomes are a result of multiple factors. First and foremost, the diagnosis of visceral malperfusion is very challenging as abdominal pain at the onset of presentation is not always present, and the serum lactate may only rise late in the process when transmural intestinal infarction has already occurred (8). Next, due to decreased tissue oxygen delivery, vasodilation initially ensues, but over time, prolonged ischemia leads to vasoconstriction which can persist long after the ischemic insult is complete (9). Lastly, the paradoxical consequences of reperfusion injury to these ischemic vascular beds resulting from the release of inflammatory cytokines and formation free oxygen radicals leading to cellular and endothelial injury, increased tissue permeability and edema. Importantly, this inflammatory response can progress systemically leading to respiratory distress syndrome and shock (3). In previous reports, an absence of distal signals along with necrotic bowel was found despite presence of a strong doppler pulse signal at the mesenteric root at the time of laparotomy. Such findings are consistent with persistent vasospasm, the pathophysiologic process observed in nonocclusive mesenteric ischemia, and is likely a principal factor in the $60 \%$ to $80 \%$ mortality (10).
Thus, minimizing the ischemic time is critical in order to prevent this cascade of devastating events. In this work, we discuss our experience with the TEVAR-first approach adopted for type A dissection with mesenteric malperfusion at Emory University (Video 1) as well as other approaches employed to address this life-threatening complication. We present the following article in accordance with the Narrative Review reporting checklist (available at https:// jovs.amegroups.com/article/view/10.21037/jovs-20-155/rc).

\section{Methods}

Original and review articles on management of mesenteric malperfusion published between 1990 to 2019 were searched on PubMed database.

\section{Discussion}

TAAD complicated by mesenteric malperfusion has traditionally been addressed with emergent proximal aortic repair followed by exploratory laparotomy and bowel resection. As discussed earlier, operative mortality is high and is likely a result of persistent distal branch vessel ischemia either due to re-entry tears, persistent false lumen flow or branch vessel involvement. Several approaches have been suggested by different groups across the world to address this problem. A group from Japan have utilized a simultaneous approach whereby a laparotomy to perform intestinal resection is done along with temporary SMA perfusion via a side graft or separate cannula through the ostium during cardiopulmonary bypass. Proximal aortic repair is then performed followed by SMA reperfusion via SMA plasty or bypass graft from common iliac artery $(11,12)$. Totsugawa et al. reported using a column of polymyxin B-immobilized fiber (PMX-DHP) during cardiopulmonary bypass to combat reperfusion injury (13). In 1996, the group at the University of Michigan adopted a new approach whereby percutaneous endovascular revascularization is performed by the interventional radiology and achieved vis fenestration and/or stenting of the critically malperfused abdominal organs or extremities, followed by open proximal aortic repair. These procedures are performed in hybrid suites with the ability to perform both endovascular and open procedures. Treatable malperfusion syndrome is confirmed angiographically by a significant pressure gradient ( $>15 \mathrm{mmHg}$ ) between ascending aortic true lumen and a branch artery. Fenestration and stenting 
are performed via percutaneous access by creating a tear in the dissection flap to equalize the blood pressure and permit flow between the true and false lumens. It was most frequently created above the celiac artery for visceral malperfusion and below the lowest renal artery for iliac artery malperfusion. Following correction of dynamic obstruction by aortic fenestration/stenting, if the pressure gradient between the ascending aorta and a dissected branch vessel remains $>15 \mathrm{mmHg}$, bare stents are placed into the true lumen of the branch vessel beyond the terminal extent of the dissection (14-16). This group has mastered this approach over two decades and has achieved a low mortality rate for open aortic repair after interventional fenestration and stenting of $3.7 \%$.

We extrapolated our previous experience with the use of thoracic endovascular aortic repair (TEVAR) in acute type $B$ dissection and formulated a hybrid approach which was first employed in 2014. The Emory protocol, published in 2019, utilizes TEVAR to restore normal flow in the downstream aorta, either at the time of emergent central aortic repair or before undertaking the proximal aortic replacement based on the hemodynamic stability. In cases of rupture or hemodynamic instability, a pre bypass axillarybifemoral bypass graft is performed followed immediately by central aortic repair with antegrade deployment of a TEVAR graft at the time of hypothermic circulatory arrest. On the other hand, in hemodynamically stable patients without pericardial effusion, signs of aortic rupture or ongoing chest pain, we perform TEVAR first with angiographic confirmation of adequate celiac and SMA perfusion followed by a brief period of resuscitation in ICU to achieve correction of lactic acidosis and resolution of abdominal pain followed by delayed proximal aortic repair within 24 hours (8).

The diagnosis of mesenteric malperfusion is made based on a combination of patient history and physical exam findings, lab values and radiographic findings on initial CT imaging. Patients may complain of abdominal pain in addition to chest pain. On exam, they have clinical findings consistent with an acute abdomen or pain out of proportion to their exam, with multiple patients also having hematochezia. Elevated serum lactate levels, abnormal liver function tests and coagulation profiles are noted on their admission laboratory values and increase suspicion of the diagnosis. Finally, on the cross-sectional contrast imaging, all patients have evidence of severely compromised flow to the visceral segment of the abdominal aorta, including high-grade compression or complete obstruction of the true lumen at the level of celiac and superior mesenteric arteries. Often these patients may also have evidence of concurrent renal, iliofemoral or spinal malperfusion as seen by findings of elevated serum BUN/creatinine, decreased or absent peripheral pulses and loss of sensory or motor function in the lower extremities. Patients who are selected to receive an antegrade TEVAR during proximal aortic repair undergo the procedure in a standard operating room without fluoroscopy. After initiation of hypothermic circulatory arrest and arch resection but before construction of the arch anastomosis, a thoracic endoprosthesis is deployed in the descending aorta just distal to the left subclavian artery. The arch anastomosis is performed, incorporating the endograft into the distal arch suture line.

For the TEVAR-first treatment algorithm, the initial endovascular procedure is performed in a hybrid operating room with fluoroscopy. Bilateral femoral arterial access is obtained and intravascular ultrasound (IVUS) is used to confirm true lumen wire access. Based on preoperative imaging and IVUS measurements of the adventitia-toadventitia diameter of the dissected aorta at the distal edge of the left subclavian artery, thoracic endografts are selected with $<10 \%$ oversizing. Thoracic endografts are deployed in zone 3 within $2 \mathrm{~cm}$ of the left subclavian artery. No attempt is made to cover the primary intimal tear because the goal of the TEVAR procedure is solely to expand the true lumen and increase arterial inflow to the distal thoracic and abdominal aorta and visceral segment. Following deployment of the initial endograft, IVUS is repeated to confirm true lumen expansion and adequate branch vessel perfusion. Additional stents are added as needed, and aortic branch vessel stenting is performed until normal perfusion of the visceral aortic segment is confirmed by abdominal aortography and femoral pressures matched right radial artery pressures. Patients are then returned to the intensive care unit and undergo serial abdominal exams with aggressive intravenous fluid resuscitation. Ideally the patients are also extubated, and the overall goal is to normalize the serum lactate before open proximal aortic replacement. It is also important to maintain adequate hemodynamic control during this time period with wellcontrolled systolic blood pressures $<120-140 \mathrm{mmHg}$.

Following this 24-hour resuscitative period, the patient is brought back to the operating room for definitive proximal aortic repair. The visceral organs remain at risk for ischemia reperfusion injury during this time and therefore every attempt is made to minimize the circulatory arrest and bypass times. The majority of these patients 


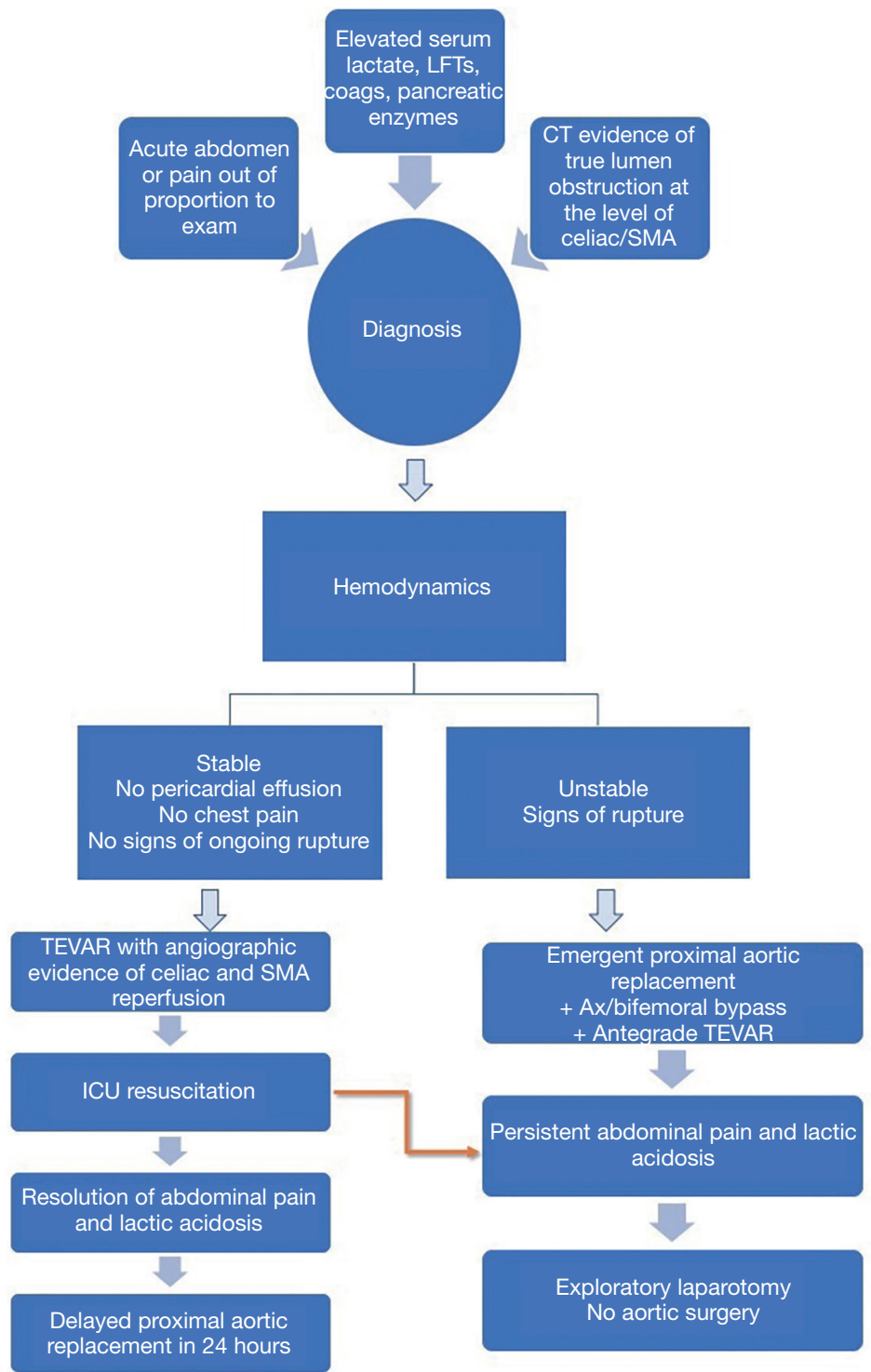

Figure 1 Emory algorithm for management of mesenteric malperfusion. TEVAR, thoracic endovascular aortic repair; SMA, superior mesenteric artery.

undergo hemiarch replacement and the indication for aortic root replacement includes the presence of concomitant aneurysmal disease of the sinus segments with diameter $>4.5 \mathrm{~cm}$ or extensive sinus segment destruction. The indications for total arch replacement include a primary or large secondary intimal tear in the arch, aneurysmal arch disease with a diameter $>4.5 \mathrm{~cm}$, or extensive tissue destruction that could not be safely reconstructed with a hemiarch replacement. Our algorithmic approach is illustrated in Figure 1.

Overall mortality in our initial series with this approach was $30 \%$, as compared to $69 \%$ with the traditional approach. Neither post-operative bowel ischemia or intractable acidosis, known to be independent risk factors for mortality, were observed in our cohort. In addition, a trend was observed towards a reduced need for dialysis in patients treated with the TEVAR first strategy as compared to those treated with the traditional approach.

We believe that TEVAR first approach can also be used as a bridge to decision making in patients who present with advanced mesenteric ischemia and are unlikely to survive. Patients who have intractable acidosis or unresectable bowel 
necrosis despite TEVAR are unlikely to survive proximal aortic replacement under hypothermic circulatory arrest and thus can be spared an invasive procedure which utilizes significant resources. On the other hand, we strongly favor limiting the waiting time between TEVAR and proximal aortic repair to no longer than 24-48 hours to minimize the risk of inadvertent aortic rupture.

TEVAR successfully relieves dynamic obstruction of the true lumen and rapidly restores perfusion to branch vessels. Moreover, it offers the ability for intra-operative, real time, confirmation of visceral reperfusion via angiography and selective blood pressure measurements which would not be possible during a proximal aortic repair where there is minimal access to the abdomen. Additional branch vessel stenting can be performed as needed, to restore perfusion in cases of static obstruction from false lumen thrombosis.

Initially in our experience, we performed antegrade TEVAR during the proximal aortic repair as described above, but these patients were also noted to have a poor outcome due to persistent metabolic acidosis and gastrointestinal sepsis. Even though quicker restoration of visceral perfusion via this technique was evident, it does not prevent the insult resulting from circulatory arrest in an already ischemic vascular bed. In addition, circulatory arrest and cardiopulmonary bypass times are inevitably increased with no real time confirmation of actual flow restoration. Thus, in patients with TAAD complicated by both mesenteric malperfusion as well as hemodynamic instability, it may be reasonable to consider deploying a TEVAR graft prior to opening the chest for proximal aortic repair in order to reduce the ischemic period while minimizing the time of circulatory arrest needed for arch reconstruction.

Some centers use advanced endovascular techniques with fenestration combined with branch vessel stenting followed by delayed aortic repair. This technique also has excellent outcomes, but we feel that its use is restricted to highly specialized centers who have advanced interventional radiology resources. TEVAR, on the other hand, is a commonly employed technique across several centers in treatment of type B dissections by both cardiothoracic and vascular surgeons, hence the familiarity could lead to a more widespread adoption in centers who may not have access to advanced interventional radiologists on a round-the-clock basis.

One limitation of TEVAR lies in its inability to simultaneously address cerebral malperfusion. Acute type A dissection complicated by a combination of cerebral malperfusion with visceral or iliac malperfusion is challenging and any sign of cerebral malperfusion with a neurological deficit should prompt immediate proximal aortic repair. An additional disadvantage of TEVAR first involves the vulnerable resuscitative period following correction of malperfusion where there is a constant risk of aortic rupture. Patients should thus be carefully monitored for any signs of hemodynamic instability, pericardial effusion, or chest pain and ideally, proximal aortic repair should be performed within 24 hours.

\section{Conclusions}

In conclusion, immediate proximal aortic repair for TAAD complicated by mesenteric malperfusion is associated with poor clinical outcomes, Alternate approaches with use of endovascular techniques to first correct mesenteric malperfusion prior to open aortic replacement are increasingly being employed. Delayed proximal aortic replacement after initial TEVAR has preliminarily been demonstrated to be a straightforward and reliable approach to this condition while providing for immediate confirmation of flow restoration to the gut vessels. Larger and multi-center studies investigating the ultimate merit of this approach are warranted.

\section{Acknowledgments}

Funding: None.

\section{Footnote}

Provenance and Peer Review: This article was commissioned by the Guest Editors (Roberto Di Bartolomeo, Davide Pacini and Mohamad Bashir) for the series "The 10th Postgraduate Course on 'Surgery of the Thoracic Aorta' in Bologna" published in Fournal of Visualized Surgery. The article has undergone external peer review.

Reporting Checklist: The authors have completed the Narrative Review reporting checklist. Available at https:// jovs.amegroups.com/article/view/10.21037/jovs-20-155/rc

Conflicts of Interest: All authors have completed the ICMJE uniform disclosure form (available at https://jovs. amegroups.com/article/view/10.21037/jovs-20-155/coif). The series "The 10th Postgraduate Course on 'Surgery of the Thoracic Aorta' in Bologna" was commissioned by the editorial office without any funding or sponsorship. 
BGL reports personal fees from Medtronic Inc., outside the submitted work. The authors have no other conflicts of interest to declare.

Ethical Statement: The authors are accountable for all aspects of the work in ensuring that questions related to the accuracy or integrity of any part of the work are appropriately investigated and resolved.

Open Access Statement: This is an Open Access article distributed in accordance with the Creative Commons Attribution-NonCommercial-NoDerivs 4.0 International License (CC BY-NC-ND 4.0), which permits the noncommercial replication and distribution of the article with the strict proviso that no changes or edits are made and the original work is properly cited (including links to both the formal publication through the relevant DOI and the license). See: https://creativecommons.org/licenses/by-nc-nd/4.0/.

\section{References}

1. Berretta P, Patel HJ, Gleason TG, et al. IRAD experience on surgical type A acute dissection patients: results and predictors of mortality. Ann Cardiothorac Surg 2016;5:346-51.

2. Berretta P, Trimarchi S, Patel HJ, et al. Malperfusion syndromes in type A aortic dissection: what we have learned from IRAD. J Vis Surg 2018;4:65.

3. Goldberg JB, Lansman SL, Kai M, et al. Malperfusion in Type A Dissection: Consider Reperfusion First. Semin Thorac Cardiovasc Surg 2017;29:181-5.

4. Kamman AV, Yang B, Kim KM, et al. Visceral Malperfusion in Aortic Dissection: The Michigan Experience. Semin Thorac Cardiovasc Surg 2017;29:173-8.

5. Leontyev S, Légaré JF, Borger MA, et al. Creation of a Scorecard to Predict InHospital Death in Patients Undergoing Operations for Acute Type A Aortic Dissection. Ann Thorac Surg 2016;101:1700-6.

doi: 10.21037/jovs-20-155

Cite this article as: Kalra K, Leshnower BG, Chen EP. Narrative review of the thoracic endovascular aortic repairfirst approach for mesenteric malperfusion in type A aortic dissection. J Vis Surg 2022;8:2.
6. Pacini D, Leone A, Belotti LM, et al. Acute type A aortic dissection: significance of multiorgan malperfusion. Eur J Cardiothorac Surg 2013;43:820-6.

7. Fann JI, Sarris GE, Mitchell RS, et al. Treatment of patients with aortic dissection presenting with peripheral vascular complications. Ann Surg 1990;212:705-13.

8. Leshnower BG, Keeling WB, Duwayri YM, et al. The "thoracic endovascular aortic repair first" strategy for acute type A dissection with mesenteric malperfusion: Initial results compared with conventional algorithms. J Thorac Cardiovasc Surg 2019;158:1516-24.

9. Clair DG, Beach JM. Mesenteric ischemia. N Engl J Med 2016;374:959-68.

10. Brandt LJ, Feuerstadt P. Intestinal ischemia. In: Feldman M, Friedman LS, Brandt LJ. editors. Sleisenger and Fordtran's Gastrointestinal and Liver Disease 10th ed. Philadelphia: Saunders, 2016:2076-101.

11. Okada Y, Okimoto M, Katsumata M, et al. Temporary perfusion for mesenteric ischemia with acute type A aortic dissection. Ann Thorac Surg 2007;83:293-4.

12. Kato W, Fujita T, Uchida K, et al. Superior mesenteric artery plasty for type A acute aortic dissection with visceral ischemia. Gen Thorac Cardiovasc Surg 2016;64:422-4.

13. Totsugawa $T$, Ozawa $M$, Kuinose $M$, et al. Intraoperative endotoxin adsorption for visceral malperfusion complicating acute type A aortic dissection. Gen Thorac Cardiovasc Surg 2010;58:134-7.

14. Yang B, Rosati CM, Norton EL, et al. Endovascular Fenestration/Stenting First Followed by Delayed Open Aortic Repair for Acute Type A Aortic Dissection With Malperfusion Syndrome. Circulation 2018;138:2091-103.

15. Patel HJ, Williams DM, Dasika NL, et al. Operative delay for peripheral malperfusion syndrome in acute type A aortic dissection: A long-term analysis. J Thorac Cardiovasc Surg 2008;135:1288-95.

16. Deeb GM, Williams DM, Bolling SF, et al. Surgical delay for acute type A dissection with malperfusion. Ann Thorac Surg 1997;64:1669-75. 